XXXX

\title{
Quantum Quench and Double Trace Couplings
}

\author{
Pallab Basu ${ }^{(a)}$, Diptarka Das ${ }^{(b)}$, Sumit R. Das ${ }^{(b)}$ and Krishnendu Sengupta ${ }^{(c)}$ \\ (a) International Center for Theoretical Sciences, Tata Institute of Fundamental Research, Bangalore \\ 560012, INDIA \\ ${ }^{(b)}$ Department of Physics and Astronomy, University of Kentucky, Lexington, KY 40506, USA \\ (c) Department of Theoretical Physics, Indian Association for the Cultivation of Science, Jadavpur, \\ Kolkata 700032, INDIA \\ E-mail: pallabbasu@gmail.com, diptarka.das@uky.edu, das@pa.uky.edu, \\ ksengupta1@gmail.com
}

ABstract: We consider quantum quench by a time dependent double trace coupling in a strongly coupled large $\mathrm{N}$ field theory which has a gravity dual via the AdS/CFT correspondence. The bulk theory contains a self coupled neutral scalar field coupled to gravity with negative cosmological constant. We study the scalar dynamics in the probe approximation in two backgrounds: AdS soliton and AdS black brane. In either case we find that in equilibrium there is a critical phase transition at a negative value of the double trace coupling $\kappa$ below which the scalar condenses. For a slowly varying homogeneous time dependent coupling crossing the critical point, we show that the dynamics in the critical region is dominated by a single mode of the bulk field. This mode satisfies a Landau-Ginsburg equation with a time dependent mass, and leads to Kibble Zurek type scaling behavior. For the AdS soliton the system is non-dissipative and has $z=1$, while for the black brane one has dissipative $z=2$ dynamics. We also discuss the features of a holographic model which would describe the non-equilibrium dynamics around quantum critical points with arbitrary dynamical critical exponent $z$ and correlation length exponent $\nu$. These analytical results are supported by direct numerical solutions. 


\section{Contents}

1 Introduction and Summary 1

2 The equilibrium critical point $\quad 5$

2.1 Pure $A d S_{d+2}$

$2.2 \quad A d S_{d+2}$ soliton 7

2.2.1 Effect of non-linearity 8

$2.3 A d S_{d+2}$ Black Brane $\quad 10$

3 Slow Quench with a time dependent $\kappa$ in $A d S_{d+2}$ soliton background $\quad 11$

3.1 Breakdown of Adiabaticity 12

3.2 Dynamics in the critical region 13

4 Slow quench with a time dependent $\kappa: A d S_{d+2}$ black brane 14

4.1 Breakdown of Adiabaticity 14

4.2 Dynamics in The Critical Region 15

5 Numerical Results $\quad 17$

$\begin{array}{ll}5.1 \text { Soliton background } & 17\end{array}$

$\begin{array}{ll}5.2 \text { Black Brane background } & 17\end{array}$

6 Arbitrary exponents and Kibble-Zurek Scaling $\quad 19$

$\begin{array}{lll}7 & \text { Remarks } & 21\end{array}$

8 Acknowledgements $\quad 22$

9 Appendix : Validity of the small $v$ expansion $\quad 22$

\section{Introduction and Summary}

There has been a lot of interest in understanding the problem of thermal or quantum quench $[1,2]$ using gauge-gravity duality [3]. One set of works concentrate on the question of thermalization by horizon formation [4]-[8] and possible resolutions of spacelike singularities [9]. Recently there have been several studies of holographic quench which involve critical points. In [10] two of us initiated the study of holographic quench across finite temperature and finite chemical potential critical points, and found hints of a mechanism which gives rise to Kibble-Zurek scaling in critical dynamics $[1,11]$. This mechanism was confirmed for a 
zero temperature but nonzero chemical potential quantum critical point in [12]. In slightly different directions $[13,14]$ studied relaxation dynamics following a thermal quench from a broken symmetry phase and [15] studied scaling behavior of final values of observables due to a thermal quench. Quantum quench in solvable large- $\mathrm{N}$ field theories without the use of gauge-gravity duality has been studied in [16-19].

In [10] and [12] the quench was due to a homogeneous time dependent source for a scalar order parameter which translates to a time dependent Dirichlet boundary condition on the strongly self-coupled bulk scalar field. The other parameters in the theory were tuned such that in the absence of a source the theory is critical. The dynamics was then studied in the probe approximation by considering a source which is slowly varying at early and late times and which crosses zero (i.e. the location of the critical point) at some intermediate time. In this setup scaling behavior appears due to a few key facts

- At the equilibrium critical point the linearized bulk equation of motion for the scalar has a zero mode. This results in a breakdown of adiabaticity when the source becomes small characterized by a power law in the rate of change of the source $v$.

- In the critical region, and only in this region, there a new expansion for small $v$. This is an expansion in fractional powers of $v$, with exponents determined by the equilibrium critical exponents.

- In the lowest order of this expansion in fractional powers of $v$, the bulk dynamics is dominated by the zero mode. This zero mode then satisfies an ordinary differential equation which is basically the dynamics of the order parameter. In this equation, the boundary condition appears as a source term. This equation has a scaling solution displaying Kibble-Zurek scaling.

The setup in [10] and [12] involved a nonzero chemical potential and/or nonzero temperature. The background in [10] is a charged black brane with a neutral self coupled scalar [20], while that in [12] is an AdS soliton with a constant gauge field and a self coupled scalar - a variation of the setup of [21]. In both cases the resulting dynamics of the order parameter is non-relativistic with dynamical critical exponent $z=2$, even though the underlying bulk dynamics is relativistic. It is possible that the zero temperature limit of the setup of [10] may lead to a $z=1$ dynamics. However the zero temperature limit the phase transition found in [20] and probed in [10] becomes a Berezinski-Kosterlitz-Thouless transition and we were not able to get any analytic handle on the dynamics.

So far all studies of quantum or thermal quench using holographic methods have dealt with time dependent external sources. A useful example to keep in mind is a magnet in the presence of a time dependent magnetic field. Critical dynamics can be then studied by tuning the temperature to the critical value. In a Landau-Ginsburg language this corresponds to a time dependent inhomogeneous term in the LG equation. In many situations, this is not a natural thing to do. For example in a superconductor an external source for the 
order parameter is not very natural, though it can be achieved by considering junctions. On the other hand, the standard tuning parameter in a critical transition is the term in a LG hamiltonian which is quadratic in the order parameter: we will call this a LG mass term.

In this paper we initiate the study of quench by such a time dependent LG mass using holographic techniques. While studying holographic quench with time dependent external source is straightforward because it maps to a time dependent boundary condition for the dual field, a time dependent LG mass quench would involve addition of a double trace deformation with a time dependent coefficient, $\kappa(t)$. As is well known this implies a modified boundary condition for the bulk scalar [25]. In equilibrium [22] found that for a class of scalar potentials, there is a critical phase transition at $\kappa=\kappa_{c}$ where $\kappa_{c}<0$. Naively, from the field theory viewpoint, a deformation with negative $\kappa$ appears to lead to an instability. However it has been shown in [22] and [23] this is not necessarily correct - typically there is a stable ground state with scalar hair for $\kappa<\kappa_{c}$. For vanishing temperature and vanishing chemical potential $\kappa_{c}=0$, while for a nonzero temperature (i.e a black hole background) $T$ one has $\kappa_{c} \propto T$. In the following we will show, not surprisingly, that there is a similar transition when the background is a $A d S$ soliton.

We consider the simplest situation where such a transition occurs. The bulk action is given in $L_{A d S}=1$ units

$$
S=\int d^{d+2} x \sqrt{g}\left[\frac{1}{8 \pi G_{N}}(R+d(d+1))-\frac{1}{\lambda}\left((\nabla \phi)^{2}+m^{2} \phi^{2}+V(\phi)\right)\right]
$$

where $\phi$ is a neutral bulk scalar. We will consider the limit $\lambda \gg G_{N}$ so that the scalar can be treated as a probe field whose dynamics does not affect the gravity background. We will consider potentials $V(\phi)$ which have a power series expansion in $\phi$. As will become clear soon, the critical behavior is determined by the leading nonlinearity in $V(\phi)$, so it would be sufficient to consider monomials.

First we study the equilibrium transition in three such backgrounds. The first is pure $A d S_{d+2}$, which is the relevant geometry when all the spatial directions are noncompact,

$$
d s^{2}=r^{2}\left(-d t^{2}+d \vec{x}^{2}+d w^{2}\right)+\frac{d r^{2}}{r^{2}}
$$

The second is a $A d S_{d+2}$ soliton which is the relevant geometry when one of the spatial directions, $w$ is compact with some radius $R_{0}$,

$$
\begin{gathered}
d s^{2}=r^{2}\left(-d t^{2}+d \vec{x}^{2}+f_{s}(r) d u^{2}\right)+\frac{d r^{2}}{r^{2} f_{s}(r)} \\
f_{s}(r)=1-\left(\frac{r_{0}}{r}\right)^{d+1}, \quad r_{0}=\frac{4 \pi}{(d+1) R_{0}}
\end{gathered}
$$

The third is a $A d S_{d+2}$ black brane with all boundary directions non-compact. The metric is

$$
\begin{aligned}
d s^{2} & =-r^{2} f_{b}(r) d t^{2}+r^{2}\left(d \vec{x}^{2}+d w^{2}\right)+\frac{d r^{2}}{r^{2} f_{b}(r)} \\
f_{b}(r) & =1-\left(\frac{\bar{r}_{0}}{r}\right)^{d+1}, \quad \bar{r}_{0}=\frac{4 \pi T}{(d+1)}
\end{aligned}
$$


In all these cases the asymptotic form of the solution for the scalar has the form

$$
\phi(r, \vec{x}, t, u) \rightarrow r^{-\Delta_{-}} \mathcal{A}(\vec{x}, u, t)\left(1+O\left(1 / r^{2}\right)\right)+r^{-\Delta_{+}} \mathcal{B}(\vec{x}, u, t)\left(1+O\left(1 / r^{2}\right)\right)
$$

provided the solution becomes small near the boundary. In (1.5)

$$
\Delta_{ \pm}=(d+1) / 2 \pm \sqrt{(d+1)^{2} / 4+m^{2}}
$$

We will work in the mass range $-(d+1)^{2} / 4 \leq m^{2} \leq-(d+1)^{2} / 4+1$ so that we have two possible quantizations [24]: the standard quantization with $\mathcal{A}$ as the source and the alternative quantization with $\mathcal{B}$ as the source. The dimensions of the dual operator $\mathcal{O}$ in these two quantizations are $\Delta_{+}$and $\Delta_{-}$respectively.

It is also possible to impose boundary conditions

$$
\mathcal{B}(\vec{x}, u, t)=\kappa(\vec{x}, u, t) \mathcal{A}(\vec{x}, u, t)
$$

As is well known, this corresponds to addition of a term [25]

$$
\int d^{d+1} x \kappa \mathcal{O}^{2}
$$

to the field theory action.

We will first show explicitly for suitable potentials that for a constant $\kappa$ all these backgrounds admit critical points. For $A d S_{d+2}$ the critical value is $\kappa=0$ : for $\kappa<0$ there is a nontrivial solution of the equations of motion which is regular everywhere and satisfies the specified boundary conditions. This means that for the dual operator $\langle\mathcal{O}\rangle \neq 0$. Near the critical point we verify that

$$
\langle\mathcal{O}\rangle \sim(-\kappa)^{\Delta_{-} /\left(\Delta_{+}-\Delta_{-}\right)}
$$

as required by scale symmetry. The scaling behavior is independent of the nature of the potential whose properties enter only in the overall coefficient.

For the $A d S_{d+2}$ soliton as well as the $A d S_{d+2}$ black brane, the critical value of $\kappa$ is at some finite value $\kappa_{c}<0$ and the condensate appears for $\kappa<\kappa_{c}$. This is shown by a direct numerical solution. It turns out that the value of $\kappa_{c}$ can be determined analytically in closed form, following the treatment of [22] and is independent of the nature of the non-linearity. As is usual in such situations, there is a zero mode of the linearized equation at $\kappa=\kappa_{c}$ : here the zero mode has a closed form in terms of hypergeometric functions. We verify that our numerical solution for $\phi^{4}$ and $\phi^{6}$ potentials agrees with this. The critical exponent can be also determined analytically. When the leading nonlinearity is $\phi^{n+1}$, one gets

$$
\langle\mathcal{O}\rangle \sim\left(\kappa_{c}-\kappa\right)^{1 /(n-1)}
$$

Note that in standard notation the critical exponent $\beta$ is given by

$$
\beta=1 /(n-1)
$$


Our numerical results are consistent with the behavior (1.10). We also verify numerically that the critical behavior is indeed determined by the leading non-linearity.

We then consider the response of the system to a time dependent but homogeneous $\kappa(t)$ for the $A d S_{d+2}$ soliton and $A d S_{d+2}$ black brane backgrounds, staying in the probe approximation. For these backgrounds, the radius of the compact dimension (for the soliton) or the temperature (for the black brane) provides a scale, so that we can meaningfully talk about slow and fast quenches. We concentrate on slow quench starting deep in the ordered phase, crossing the critical point $\kappa_{c}$ at some intermediate point and asymptoting to some other constant value at late times. Following the lines of $[10,12]$ we study the breakdown of adiabaticity and show that in a way similar to these works the critical region is characterized by an expansion in fractional powers of the rate and by the dominance of the zero mode. For a fast quench we expect a chaotic behavior to set in [27]. Unlike these previous works, the function $\kappa(t)$ now appears as a time dependent mass term in the effective LG dynamics of the zero mode and hence the order parameter. This is consistent with the fact that in the field theory, $\kappa(t)$ is indeed the coefficient of $\mathcal{O}^{2}$.

The ensuing critical dynamics for the soliton and the black brane are different. For the soliton, the dynamics is relativistic and non-dissipative. This is expected since in the field theory is at zero temperature and there is no chemical potential. The dynamics in the black brane background has $z=2$ and is dissipative, as would be expected for a finite temperature situation.

Finally we solve the time evolution numerically and provide evidence for the scaling behavior discussed above.

In Section 2 we set up the equilibrium problems, show the existence of the critical point for negative constant $\kappa$ and derive the critical exponents. Sections 3 and 4 deal with quantum quench due to a time dependent $\kappa(t)$ for the soliton and black brane backgrounds respectively. In section 5 we present our numerical results. In section 6 we discuss arbitrary critical exponents $z$ and $\nu$ and the relationship of our scaling solutions with standard Kibble-Zurek scaling. Section 7 contains brief remarks and the appendix discusses the solution of a toy model which justifies some key ingredients in our discussion of section 4 .

\section{The equilibrium critical point}

In the probe approximation the only relevant equation we need to solve is the scalar field equation. For the backgrounds (1.2) or (1.3) and fields which depend only on $t$ and $r$ this equation is

$$
-\frac{1}{h(r)} \partial_{t}^{2} \phi+\frac{1}{r^{d-2}} \partial_{r}\left(r^{d} g(r) \partial_{r} \phi\right)-m^{2} r^{2} \phi-r^{2} V^{\prime}(\phi)=0
$$

where

$$
g(r)= \begin{cases}r^{2}, & \text { for } A d S_{d+2} \\ r^{2} f_{s}(r) & \text { for } A d S_{d+2} \text { soliton } \\ r^{2} f_{b}(r) & \text { for } A d S_{d+2} \text { black brane }\end{cases}
$$


and

$$
h(r)= \begin{cases}1 & \text { for } A d S_{d+2} \text { and } A d S_{d+2} \text { soliton } \\ f_{b}(r) & \text { for } A d S_{d+2} \text { black brane }\end{cases}
$$

We first need to find static solutions of (2.1) which are regular in the interior and which satisfy the boundary condition (1.7) at the boundary (with constant $\mathcal{A}, \mathcal{B}, \kappa$ ).

\subsection{Pure $A d S_{d+2}$}

For pure $A d S_{d+2}$ and a $\phi^{4}$ potential regularity means that the value of the field at $r=0$ is fixed to the attractor value

$$
\phi(r=0)_{A d S}=\sqrt{-m^{2}}
$$

To find a solution to the non-linear equation consider integrating the equation by imposing the condition at small $\epsilon$

$$
\phi(\epsilon)=\phi(r=0)_{A d S}-c \epsilon^{\Delta_{v}} \quad c>0 \quad \Delta_{v}=\sqrt{(d+1)^{2} / 4-2 m^{2}}-(d+1) / 4
$$

This form is dictated by the solution near $r=0$ where the departure from the attractor value is small so that the equation can be linearized. The solution to the full nonlinear equation may be therefore written as $\phi(r, c)$, which gives us a one parameter class of solutions. However the equation has a scaling symmetry under

$$
r \rightarrow \lambda r \quad \phi \rightarrow \phi
$$

which immediately implies that the solution satisfies

$$
\phi_{A d S}(r, c)=\phi_{A d S}\left(r c^{-1 / \Delta_{v}}, 1\right)
$$

The solution near the boundary $r=\infty$ is of the form (1.5) with constant $\mathcal{A}$ and $\mathcal{B}$ - the scaling symmetry then implies

$$
|\mathcal{A}| \sim c^{\frac{\Delta_{-}}{\Delta_{v}}} \quad|\mathcal{B}| \sim c^{\frac{\Delta_{+}}{\Delta_{v}}} \Longrightarrow|\kappa| \sim c^{\frac{\Delta_{+}-\Delta_{-}}{\Delta_{v}}}
$$

In alternative quantization, $\mathcal{A}$ is the expectation value of the dual operator and the above relations immediately implies (1.9).

The solution $\phi(r, c)$ can be found easily by numerically solving the nonlinear equation. We find that there is a nonsingular solution for any negative $\kappa$ which satisfies the above scaling behavior.

Note that the scaling argument given above does not depend on the potential being $\phi^{4}$, and is valid for an arbitrary potential $V(\phi)$. The value of the attractor is generally given by

$$
m^{2} \phi+V^{\prime}(\phi)=0
$$

and the behavior for small $r$ becomes a bit complicated, though still determined by a linear equation. Nevertheless the same scaling behavior (1.9) would follow. The numerical coefficient will of course depend on the details of the potential. 


\section{$2.2 \quad A d S_{d+2}$ soliton}

For the $A d S_{d+2}$ soliton (1.3) regularity at the tip $r=r_{0}=1$ implies that the field can attain any value $\phi_{0}$ at $r=r_{0}$ while the derivative is given by

$$
\frac{d \phi}{d r}\left(r=r_{0}\right)=\frac{1}{d+1}\left[m^{2} \phi_{0}+V^{\prime}\left(\phi_{0}\right)\right]
$$

The static solution may be now obtained by starting at some $\phi_{0}$ and integrating out to $r=\infty$. As we will see below, straightforward numerical integration then shows that a nontrivial regular solution exists only when $\kappa<\kappa_{c}$ where the critical value $\kappa_{c}$ is a negative number to be determined shortly.

In the rest of the paper we will use $r_{0}=1$ units

As is usual in such cases (e.g. for holographic superconductors [26] ) the trivial solution with $\phi=0$ is in fact unstable for $\kappa<\kappa_{c}$. To see this, let us write the linearized equation of motion as

$$
\begin{aligned}
-\partial_{t}^{2} \phi & =\tilde{\mathcal{D}} \phi \\
\tilde{\mathcal{D}} & \equiv-r^{2-d} \frac{\partial}{\partial r}\left(r^{d+2} f_{s}(r) \frac{\partial}{\partial r}\right)+m^{2} r^{2}
\end{aligned}
$$

This equation can be cast into a Schrodinger form by changing coordinates to $\rho$

$$
\rho(r)=\int_{r}^{\infty} \frac{d s}{s^{2} f_{s}^{1 / 2}(s)}
$$

and redefining the field to $\psi(\rho, t)$

$$
\phi(r, t)=\frac{1}{[r(\rho)]^{\frac{d}{2}-1}}\left(\frac{d \rho}{d r}\right)^{1 / 2} \psi(\rho, t)
$$

Note that

$$
\begin{aligned}
& \rho \sim 1 / r \quad r \rightarrow \infty \\
& \rho \sim \rho_{\star}+\frac{2 \sqrt{r-1}}{\sqrt{d+1}} \quad r \rightarrow 1
\end{aligned}
$$

where $\rho_{\star}$ is finite. For $d=3$ we get $\rho_{\star}=1.311$. Using the explicit form of $f_{s}(r)$ the equation (2.11) becomes

$$
-\partial_{t}^{2} \psi=\mathcal{D} \psi
$$

where

$$
\begin{aligned}
\mathcal{D} & =-\partial_{\rho}^{2}+V_{0}(\rho) \\
V_{0}(\rho) & =m^{2} r^{2}+\frac{4 d\left[(d+2) r^{2 d+2}-(d+3) r^{d+1}\right]-(d-1)^{2}}{16 r^{d-1}\left(r^{d+1}-1\right)}
\end{aligned}
$$


This operator appeared in [12] where it was shown that with boundary conditions corresponding to either standard or alternative quantization this has a positive spectrum. However with the modified boundary condition $\mathcal{B}=\kappa \mathcal{A}$ with $\kappa<0$ this is no longer true. In fact there is a specific value of $\kappa$ where the operator $\mathcal{D}$ has a zero mode. The equation $\tilde{\mathcal{D}} \phi=0$ (which is equivalent to $\mathcal{D} \psi=0$ ) is in fact the same as equation (B.1) in [22] and we can borrow the results. The solution which is regular at $r=1$ is given by

$\phi_{0}(r)=A\left(r^{-\Delta_{-}} F_{1}^{2}\left[\frac{\Delta_{-}}{d+1}, \frac{\Delta_{-}}{d+1}, \frac{2 \Delta_{-}}{d+1}, r^{-(d+1)}\right]+B r^{-\Delta_{+}} F_{1}^{2}\left[\frac{\Delta_{+}}{d+1}, \frac{\Delta_{+}}{d+1}, \frac{2 \Delta_{+}}{d+1}, r^{-(d+1)}\right]\right)$

where

$$
B=-\frac{\Gamma\left(\frac{2 \Delta_{-}}{d+1}\right)\left[\Gamma\left(1-\frac{\Delta_{-}}{d+1}\right]^{2}\right.}{\Gamma\left(2-\frac{2 \Delta_{-}}{d+1}\right)\left[\Gamma\left(\frac{\Delta_{-}}{d+1}\right)\right]^{2}} .
$$

The asymptotic expansion of this solution at $r=\infty$ can be read off trivially. Clearly the $\kappa$ for this solution is $\kappa=B$. This must be the critical value, $\kappa_{c}$ which is thus determined to be

$$
\kappa_{c}=-B\left(r_{0}\right)^{d+1-2 \Delta_{-}}
$$

where we have restored factors of $r_{0}$. The zero mode $\phi_{0}$ will play a key role in what follows.

For $\kappa<\kappa_{c}$ the operator has negative eigenvalues which implies that the trivial solution is unstable.

In the following we will also need the behavior of the lowest eigenvalue $\lambda_{0}$ of $\mathcal{D}$ for $\kappa<\kappa_{c}$. Generically one would expect that this would vanish linearly,

$$
\lambda_{0}(\kappa)=-c_{0}\left(\kappa_{c}-\kappa\right) .
$$

We have checked this numerically for $d=3$ and $m^{2}=-15 / 4$ and obtained $c_{0}=0.762589$. We also checked that $\kappa_{c}=-0.495$ which is consistent with (2.19) and 2.18). This behavior will be important in the dynamics.

\subsubsection{Effect of non-linearity}

We now consider the effect of non-linearity in the static solution. Consider a $Z_{2}$ invariant potential of the form ${ }^{1}$

$$
V(\phi)=\sum_{q=2}^{\infty} \lambda_{q} \phi^{2 q} .
$$

For simplicity we will assume all the $\lambda_{q}$ 's to be positive. We want to find solutions of the full nonlinear equation with specified boundary conditions at $r=\infty$ and regular in the interior. Such solutions can be constructed by numerical integration starting with a given value of $\phi_{0}$ and obtaining the solution $\phi\left(r ; \phi_{0}\right)$ from which the leading and subleading terms in the asymptotic expansion, $\mathcal{A}$ and $\mathcal{B}$ can be calculated, thus determining $\kappa\left(\phi_{0}\right)$. In all the cases

\footnotetext{
${ }^{1}$ See [31] for a discussion of scalar effective potential in $A d S$ background.
} 
we have studied, the solution is trivial for any $\kappa>\kappa_{c}$ while for $\kappa<\kappa_{c}$ there is a nontrivial solution, leading to a nonzero order parameter in the boundary theory. It is expected (and we can also verify numerically) that $\kappa_{c}$ is only a function of $m^{2}$ and it is independent of $\lambda_{q}$. Furthermore, as is usual in mean field theory, the critical exponent is determined by the leading nonlinearity. For example, if lowest nonvanishing term is $V(\phi)=\frac{1}{4} \phi^{4}$ then one expects

$$
\langle\mathcal{O}\rangle_{\text {soliton }} \sim\left(\kappa_{c}-\kappa\right)^{1 / 2}
$$

This is standard mean field behavior. The exponent should not be affected by the presence of nonvanishing $\lambda_{q}$ with $q>2$.

Figure (1) shows the result of a numerical solution of the static equations of motion for $d=3, m^{2}=-15 / 4$ for two potenitals : (i) $\lambda_{2}=1$ with all the other $\lambda_{q}$ vanishing and (ii) $\lambda_{2}=1, \lambda_{3}=20$ with the other $\lambda_{q}$ vanishing

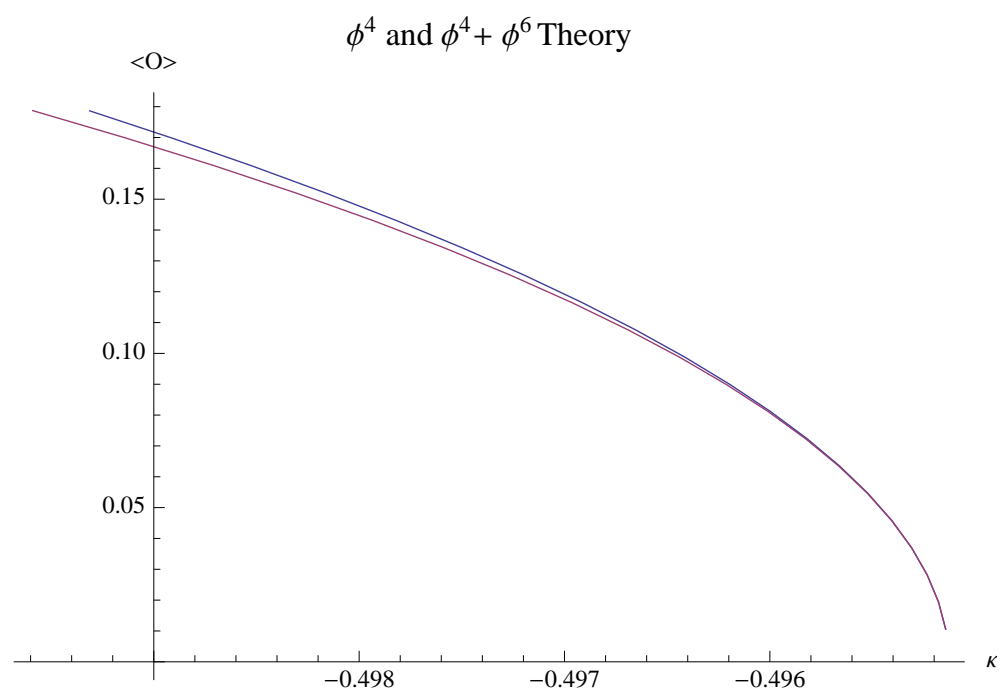

Figure 1. The order parameter as a function of $\kappa$ for $\phi^{4}$ (blue) and $\phi^{4}+\phi^{6}$ (red) theory. The critical value is around -0.495129

The critical coupling $\kappa_{c}$ is found to be $\kappa_{c}=-0.495129$ which is the same for both potentials and consistent with (2.18) and (2.19) for this value of $d, m^{2}$. Clearly the behavior of the order parameter near the critical point is the same for both potentials while the behavior differs far away from the critical point. Figure (2) shows the determination of the critical exponent for bth potentials.

If the leading non-vanishing non-linear term is of $O\left(\phi^{n+1}\right)$, i.e. $V(\phi)=\frac{1}{n+1} \phi^{n+1}+\cdots$, then we get,

$$
\langle\mathcal{O}\rangle_{\text {soliton }} \sim\left(\kappa_{c}-\kappa\right)^{\beta}
$$

where $\beta=\frac{1}{(n-1)}$. This is standard mean field multicritical behavior (for a numerical verification see Fig 4 ). 


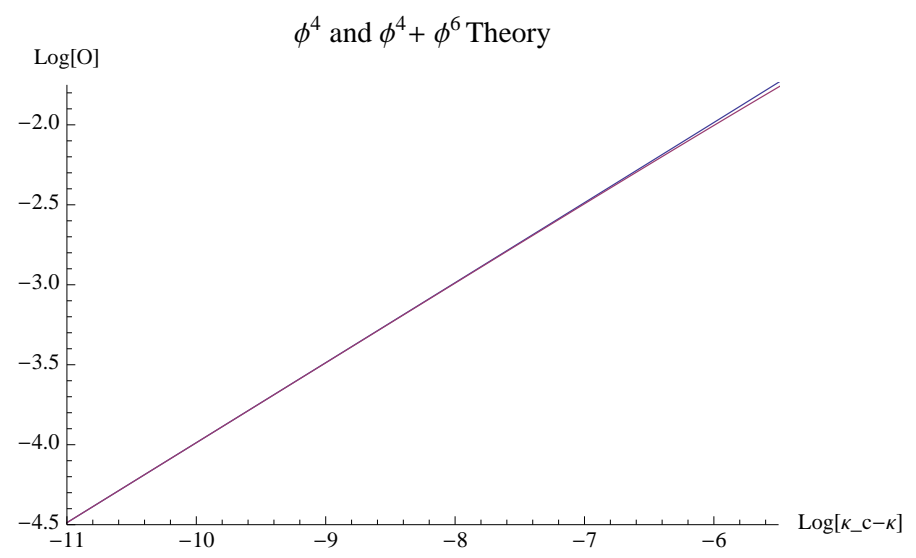

Figure 2. Plot of $\log \langle\mathcal{O}\rangle$ versus $\log \left(\kappa_{c}-\kappa\right)$ for $\phi^{4}$ (blue) and $\phi^{4}+\phi^{6}$ (red) theory. The fit for blue line is, $\log \langle\mathcal{O}\rangle=1.01802+0.500572 \log \left(\kappa_{c}-\kappa\right)$ and that for the red line is $\log \langle\mathcal{O}\rangle=0.96698+$ $0.495077 \log \left(\kappa_{c}-\kappa\right)$

The critical exponent in fact follows from the equation itself. In terms of the redefined field $\psi(\rho, t)$ ( see Eqs. (2.11) - (2.16)), the static equation of motion is

$$
\mathcal{D} \psi+G(\rho) \psi^{n}=0
$$

where

$$
G(\rho) \equiv \frac{r^{2-d}}{f_{s}(r)^{1 / 2}}
$$

Near $\kappa=\kappa_{c}$ the solution itself is small and may be expanded as

$$
\psi(\rho ; \kappa)=\epsilon^{\beta}\left(\psi^{(0)}(\rho)+\epsilon \psi^{(1)}(\rho)+\epsilon^{2} \psi^{(2)}(\rho)+\cdots\right)
$$

where

$$
\epsilon \equiv\left(\kappa_{c}-\kappa\right)
$$

where the number $\beta$ has to be determined by substituting the expansion in (2.25) and equating terms order by order in $\epsilon$. This may be easily seen to determine $\beta=\frac{1}{(n-1)}$.

\section{3 $A d S_{d+2}$ Black Brane}

The equilibrium solutions for the $A d S_{d+2}$ black brane are identical to those for the $A d S_{d+2}$ soliton with the replacement $r_{0} \rightarrow \bar{r}_{0}$. This is clear from the full equation (2.1) and the form of the functions $f_{b}(r)$ and $f_{s}(r)$ in (1.3) and (1.4).

However, the passage to the Schrodinger form of the equations is different, which leads to a different dynamics. As explained below, it is useful to use Eddington-Finkelstein coordinates which are regular at the horizon,

$$
u=\bar{\rho}-t \quad d \bar{\rho}=-\frac{d r}{r^{2} f_{b}(r)}
$$




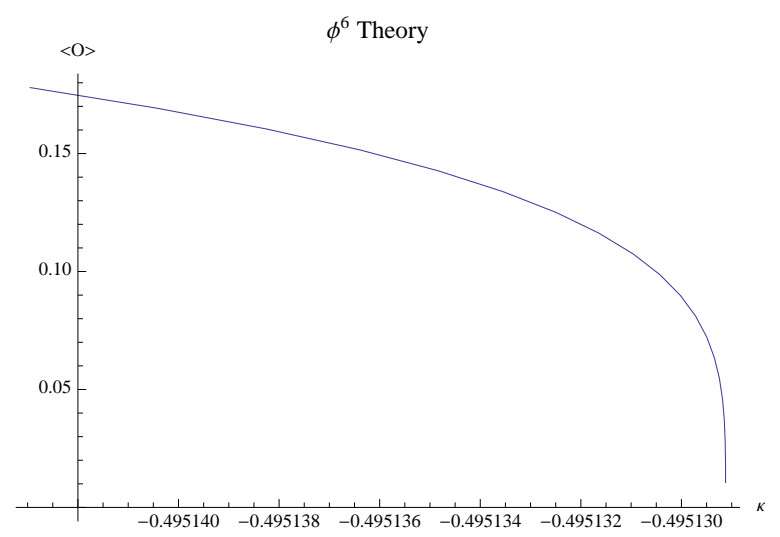

Figure 3. The order parameter as a function of $\kappa$ for $\phi^{6}$ theory. The critical value is around 0.495129 .

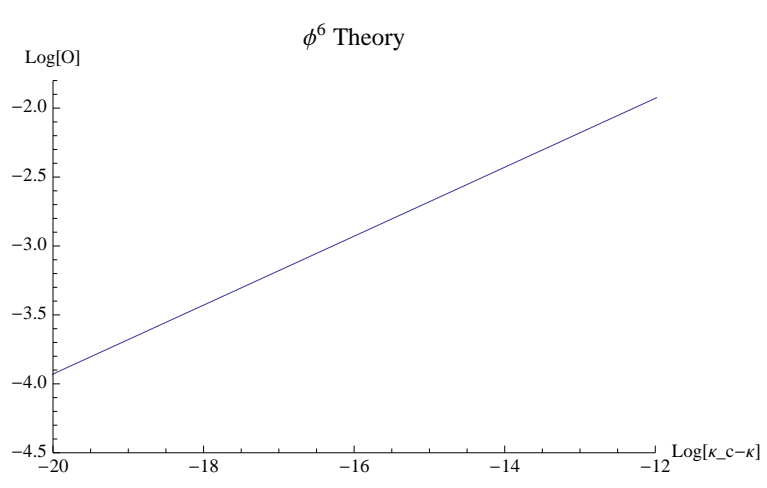

Figure 4. Plot of $\log \langle\mathcal{O}\rangle$ versus $\log \left(\kappa_{c}-\kappa\right)$ for $\phi^{6}$ theory. The fit is $\log \langle\mathcal{O}\rangle=1.07161+$ $0.250041 \log \left(\kappa_{c}-\kappa\right)$

so that the metric becomes

$$
d s^{2}=r^{2}\left(d \vec{x}^{2}+d w^{2}\right)-2 d u d r-r^{2} f_{b}(r) d u^{2}
$$

In terms of fields

$$
\chi(u, \bar{\rho})=r^{d / 2} \phi(r, t)
$$

The full equation of motion becomes

$$
-2 \partial_{u} \partial_{\bar{\rho}} \chi=\mathcal{P} \chi+\bar{G}(\bar{\rho}) \chi^{3}
$$

where

$$
\begin{aligned}
\mathcal{P} & \equiv-\partial_{\bar{\rho}}^{2}+\bar{V}_{0}(\bar{\rho}) \\
\bar{V}_{0}(\bar{\rho}) & \equiv r^{2} f_{b}(r)\left[\frac{d}{2} r \partial_{r} f_{b}(r)+\frac{d(d+2)}{4} f_{b}(r)+m^{2}\right] \\
\bar{G}(\bar{\rho}) & \equiv \frac{f_{b}(r)}{r^{d-2}}
\end{aligned}
$$

In the following we will use this form of the equations of motion to examine the dynamics.

The discussion of multicritical points is exactly the same as that in the $A d S$ soliton background in the previous section and will not be repeated here.

\section{Slow Quench with a time dependent $\kappa$ in $A d S_{d+2}$ soliton background}

We now study the response of the system in the $A d S_{d+2}$ soliton background to a time dependent $\kappa$ which starts off slowly at early times in the ordered phase $\kappa_{i}<\kappa_{c}$, crosses $\kappa_{c}$ and 
asymptotes at late times for some other value $\kappa_{f}>\kappa_{c}$. The details of the protocol are not important - however the manner in which the coupling crosses the critical value is relevant. We consider a quench which is linear near $\kappa \sim \kappa_{c}$, though all the considerations can be trivially extended to nonlinear quenches. For concreteness we consider the protocol

$$
\kappa(t)=\kappa_{c}+a \tanh (v t)
$$

with $v \ll 1$. Note that we are using units $r_{0}=1$ so what we really mean is that $v \ll r_{0}$.

\subsection{Breakdown of Adiabaticity}

At early times, the response of the system is adiabatic. The solution to the equation of motion (2.24) can be then obtained in an adiabatic expansion

$$
\psi(\rho, t ; \kappa)=\psi_{0}(\rho ; \kappa(t))+\epsilon \psi_{1}(\rho, t ; \kappa)+\epsilon^{2} \psi_{2}(\rho, t ; \kappa)+\cdots
$$

where the static solution is denoted by $\phi_{0}(r ; \kappa)$ and $\epsilon$ is the adiabaticity parameter. In the left hand side of equation (2.24) we now need to replace $\partial_{t} \rightarrow \epsilon \partial_{t}$ and equate terms with the same power of $\epsilon$. The $n$-th order contribution to the solution, $\phi_{n}$ satisfies a linear, inhomogeneous equation with the source being determined by the $\phi_{m}$ with $m<n$.

For the $\phi^{4}$ theory the two lowest order corrections satisfy

$$
\begin{aligned}
& {\left[\mathcal{D}+3 G(\rho) \psi_{0}^{2}\right] \psi_{1}=0} \\
& {\left[\mathcal{D}+3 G(\rho) \psi_{0}^{2}\right] \psi_{2}=-\partial_{t}^{2} \psi_{0}-3 G(\rho) \psi_{1}^{2} \psi_{0}}
\end{aligned}
$$

Note that $\psi_{n}$ for $n>0$ satisfy vanishing boundary conditions at infinity and regularity conditions in the interior. Since $\psi_{1}$ satisfies a homogeneous equation there is no nontrivial solution. The lowest order correction to the adiabatic solution is therefore $\psi_{2}$

$$
\psi_{2}(\rho, t ; \kappa)=-\int_{0}^{\rho_{\star}} d \rho^{\prime} \mathcal{G}\left(\rho, \rho^{\prime}\right) \partial_{t}^{2} \psi_{0}\left(\rho^{\prime}, \kappa(t)\right)
$$

where $\mathcal{G}\left(\rho, \rho^{\prime}\right)$ is the Green's function of the operator $\left[\mathcal{D}+3 G(\rho) \psi_{0}^{2}\right]$.

Exactly at $\kappa=\kappa_{c}$ the operator $\mathcal{D}$ has a zero mode. At this point the Green's function diverges and adiabaticity fails. As found in the previous section, the smallest eigenvalue for a $\kappa$ close to $\kappa_{c}$ is proportional to $\left(\kappa-\kappa_{c}\right)$. Furthermore we also found that $\psi_{0} \sim\left(\kappa_{c}-\kappa\right)^{1 / 2}$. Thus the lowest eigenvalue of the entire operator $\left[\mathcal{D}+3 G(\rho) \psi_{0}^{2}\right]$ is proportional to $\left(\kappa_{c}-\kappa\right)$. This gives an estimate of $\psi_{2}$ as we approach the critical point,

$$
\psi_{2} \sim \frac{1}{\kappa_{c}-\kappa(t)} \partial_{t}^{2} \sqrt{\kappa_{c}-\kappa(t)}=\frac{1}{2\left(\kappa_{c}-\kappa\right)^{3 / 2}}\left[\partial_{t}^{2} \kappa(t)+\frac{\left(\partial_{t} \kappa\right)^{2}}{\kappa_{c}-\kappa(t)}\right]
$$

The adiabatic expansion breaks down once $\psi_{2} \sim \psi_{0}$ which leads to the condition

$$
\left[\partial_{t}^{2} \kappa(t)+\frac{\left(\partial_{t} \kappa\right)^{2}}{\kappa_{c}-\kappa(t)}\right] \sim\left(\kappa_{c}-\kappa\right)^{2}
$$


For the protocol like (3.1), or any other protocol which is linear in time as it crosses $\kappa_{c}$ this leads to the estimate for the time when adiabaticity fails, $t_{a d}$

$$
t_{a d} \sim v^{-1 / 3}
$$

At this time the value of the order parameter is then

$$
\langle\mathcal{O}\rangle \sim\left(v t_{a d}\right)^{1 / 2} \sim v^{1 / 3}
$$

This analysis can be easily repeated for multicritical points - this will be discussed in detail in a separate section.

\subsection{Dynamics in the critical region}

Once adiabaticity is broken there is no power series expansion in $v$. We will now show that there is nevertheless an expansion for small $v$, but in fractional powers of $v$. To see this let us rescale

$$
\psi(\rho, t)=v^{1 / 3} \varphi(\rho, t) \quad t=v^{-1 / 3} \eta
$$

The equation of motion (??) now becomes

$$
\mathcal{D} \varphi=v^{2 / 3}\left(-\partial_{\eta}^{2} \varphi-G(\rho) \varphi^{3}\right)
$$

Now decompose the field in terms of eigenfunctions of $\mathcal{D}$

$$
\begin{aligned}
\varphi(\rho, \eta) & =\sum_{n} \chi_{n}(\rho) \xi_{n}(\eta) \\
\mathcal{D} \chi_{n} & =\lambda_{n}(\kappa) \chi_{n}
\end{aligned}
$$

The eigenvalues of course depend on the boundary conditions. We have expressed this explicitly by denoting them by $\lambda_{n}(\kappa)$. The equation (3.10) becomes

$$
\lambda_{n}(\kappa) \xi_{n}(\eta)=v^{2 / 3}\left(-\partial_{\eta}^{2} \xi_{n}-C_{m_{1}, m_{2}, m_{3}}^{n} \xi_{m_{1}} \xi_{m_{2}} \xi_{m_{3}}\right)
$$

where

$$
C_{m_{1}, m_{2}, m_{3}}^{n} \equiv \int_{0}^{\rho_{\star}} d \rho G(\rho) \varphi_{n}^{\star}(\rho) \varphi_{m_{1}}(\rho) \varphi_{m_{2}}(\rho) \varphi_{m_{3}}(\rho)
$$

In the previous section we showed explicitly that the lowest eigenvalue of $\mathcal{D}$ is of order $\left(\kappa_{c}-\kappa\right)$. In fact generically for theories with $\nu=1 / 2$

$$
\lambda_{n}(\kappa)=\lambda_{n}\left(\kappa_{c}\right)-c_{n}\left(\kappa_{c}-\kappa\right)+O\left[\left(\kappa_{c}-\kappa\right)^{2}\right] \quad c_{n}>0
$$

Using the fact that

$$
\kappa_{c}-\kappa(t) \sim-a(v t)=-a v^{2 / 3} \eta
$$

in the critical region, the equation (3-12) becomes

$$
\lambda_{n}\left(\kappa_{c}\right) \xi_{n}(\eta)=v^{2 / 3}\left(-\partial_{\eta}^{2} \xi_{n}-a c_{n} \eta \xi_{n}-C_{m_{1}, m_{2}, m_{3}}^{n} \xi_{m_{1}} \xi_{m_{2}} \xi_{m_{3}}\right)
$$


The boundary condition gives rise to a time dependent mass term in the equation for the mode functions. Recall that $\lambda_{0}\left(\kappa_{c}\right)=0$. The dominance of this zero mode for small $v$ is manifest in this equation. All the other modes are at least $O\left(v^{2 / 3}\right)$. The zero mode satisfies an effective Landau-Ginsburg dynamics,

$$
\partial_{\eta}^{2} \xi_{0}+c_{0} \eta+C_{000}^{0} \xi_{0}^{3}=0
$$

The order parameter, which is given in terms of the asymptotic behavior of the field, also satisfies this equation to lowest order. Reverting to the original variables we see that the order parameter as a function of time has the scaling behavior

$$
\langle\mathcal{O}\rangle(t ; v)=v^{1 / 3}\langle\mathcal{O}\rangle\left(t v^{1 / 3} ; 1\right)
$$

The dynamics is relativistic and, as will be discussed in a later section, consistent with $z=1$ Kibble Zurek scaling.

Once again the scaling solution for multicritical points follow along similar lines, as discussed below.

\section{Slow quench with a time dependent $\kappa: A d S_{d+2}$ black brane}

The analysis for the response to a slow quench in a black brane background is quite similar to above, but the results are rather different. We will not detail the analysis, but give the essential equations, highlighting the results. The key difference arises from the presence of a horizon in this geometry. We need to impose ingoing boundary conditions at the horizon. Equivalently, in the ingoing Eddington-Finkelstein coordinates we are using we need to impose a regularity at the horizon $r=1$ (in $\bar{r}_{0}=1$ units) [28].

The time coordinate is now $u$, so that the protocol is

$$
\kappa(u)=\kappa_{c}+a \tanh (v u)
$$

Note that on the boundary $u$ becomes the same as the usual time $t$ and in fact for any $r$ we have $\left.\partial_{t}\right|_{r}=\partial_{u} \mid r$, so that on the boundary this represents a time dependence identical to (3.1).

\subsection{Breakdown of Adiabaticity}

Let us first discuss usual critical points ( $\phi^{4}$ potential). Since the equation of motion $(2.31)$ is first order in $u$ derivatives the first order correction to the adiabatic result is non-vanishing. In the expansion

$$
\chi(\bar{\rho}, u ; \kappa)=\chi_{0}(\bar{\rho}, \kappa(u))+\epsilon \chi_{1}(\bar{\rho}, u)+\cdots
$$

the first order correction $\chi_{1}$ satisfies

$$
\left[\mathcal{P}+3 \bar{G}(\bar{\rho}) \chi_{0}^{2}\right] \chi_{1}=-2 \partial_{u} \partial_{\bar{\rho}} \chi_{0}
$$


An analysis identical to the one between equations (3.3) and (3.5) then leads to

$$
\chi_{1} \sim \frac{1}{\kappa_{c}-\kappa(u)} \partial_{u} \sqrt{\kappa_{c}-\kappa(u)}
$$

The condition $\chi_{0} \sim \chi_{1}$ then leads to the adiabaticity breaking time

$$
u_{a d} \sim v^{-1 / 2}
$$

while the expectation value of the operator at this time is

$$
\langle\mathcal{O}\rangle \sim v^{1 / 4}
$$

The extension of these results to multicritical points with the leading term in the potential being $\phi^{n+1}$ is straightforward, leading to

$$
u_{a d} \sim v^{-1 / 2} \quad\langle\mathcal{O}\rangle \sim v^{\frac{1}{2(n-1)}}=v^{\beta / 2}
$$

We will show below that these results are consistent with the general Kibble-Zurek relations.

\subsection{Dynamics in The Critical Region}

For the $\phi^{4}$ theory we first rescale

$$
\chi(\bar{\rho}, u)=v^{1 / 4} \bar{\chi}(\bar{\rho}, \eta) \quad u=v^{-1 / 2} \eta
$$

so that the equation $(2.31)$ becomes

$$
\mathcal{P} \bar{\chi}=-v^{1 / 2}\left[2 \partial_{\bar{\rho}} \partial_{\eta} \bar{\chi}+\bar{G}(\bar{\rho}) \bar{\chi}^{3}\right]
$$

Unlike the soliton, the spectrum of $\mathcal{P}$ is now continuous. Therefore the mode decomposition (3.11) is replaced by

$$
\bar{\chi}(\bar{\rho}, \eta)=\int d k \bar{\chi}_{k}(\bar{\rho} ; \kappa) \bar{\xi}^{k}(\eta)
$$

where

$$
\mathcal{P} \bar{\chi}_{k}(\bar{\rho} ; \kappa)=\bar{\lambda}(k ; \kappa) \bar{\chi}_{k}(\bar{\rho} ; \kappa)
$$

so that instead of (3.12) we get

$$
\bar{\lambda}(k ; \kappa) \bar{\xi}_{k}=-v^{1 / 2}\left[\int d k^{\prime} \bar{B}_{k k^{\prime}} \partial_{\eta} \bar{\xi}^{k^{\prime}}(\eta)+\int d k_{1} d k_{2} d k_{3} \bar{C}_{k_{1} k_{2} k_{3}}^{k} \bar{\xi}^{k_{1}} \bar{\xi}^{k_{2}} \bar{\xi}^{k_{3}}\right]
$$

where

$$
\begin{aligned}
\bar{B}_{k k^{\prime}} & =\int d \bar{\rho} \bar{\chi}_{k}(\bar{\rho}) \partial_{\bar{\rho}} \bar{\chi}_{k^{\prime}}(\bar{\rho}) \\
\bar{C}_{k_{1} k_{2} k_{3}}^{k} & =\int d \bar{\rho} \bar{G}(\bar{\rho}) \bar{\chi}_{k}(\bar{\rho}) \bar{\chi}_{k_{1}}(\bar{\rho}) \bar{\chi}_{k_{2}}(\bar{\rho}) \bar{\chi}_{k_{3}}(\bar{\rho})
\end{aligned}
$$


Since the operator $\mathcal{P}$ is related to the operator $\mathcal{D}$ in (2.11) by a similarity transformation (with the replacement $\bar{r}_{0} \rightarrow r_{0}$ ) the behavior of the eigenvalues $\bar{\lambda}(k ; \kappa)$ near $\kappa=\kappa_{c}$ is the same as that of $\lambda_{n}$ in (3.14)

$$
\bar{\lambda}(k ; \kappa)=\bar{\lambda}\left(k ; \kappa_{c}\right)-\bar{c}(k)\left(\kappa_{c}-\kappa\right)+O\left(\left(\kappa_{c}-\kappa\right)^{2}\right)
$$

and using the time dependence of $\kappa(u)$ near $\kappa_{c}$ we get

$$
\bar{\lambda}\left(k ; \kappa_{c}\right) \bar{\xi}_{k}=-v^{1 / 2}\left[a \bar{c}(k) \eta \bar{\xi}^{k}+\int d k^{\prime} \bar{B}_{k k^{\prime}} \partial_{\eta} \bar{\xi}^{k^{\prime}}(\eta)+\int d k_{1} d k_{2} d k_{3} \bar{C}_{k_{1} k_{2} k_{3}}^{k} \bar{\xi}^{k_{1}} \bar{\xi}^{k_{2}} \bar{\xi}^{k_{3}}\right]
$$

Recall that there is a zero mode at $\kappa=\kappa_{c}$ where the left hand side of (4.15) vanishes. If the spectrum of $\bar{\lambda}\left(k ; \kappa_{c}\right)$ were discrete it is clear from (4.15) that the zero mode dominates the dynamics. This is what happens for the $A d S$ soliton in the previous section. However the operator $\mathcal{P}$ with $\kappa=\kappa_{c}$ has a continuous spectrum and one has to careful. This analysis is, however, identical to that of [10].

The equation (4.15) suggests a solution which is an expansion in $v^{1 / 2}$ as follows

$$
\bar{\xi}^{k}(\eta)=\delta(k) \tilde{\xi}^{0}(\eta)+v^{1 / 2} \tilde{\xi}^{k}(\eta)+O(v)
$$

where again to lowest order in small $v$

$$
\begin{aligned}
0 & =\mathcal{B}_{00} \partial_{\eta} \tilde{\xi}^{0}(\eta)+a \bar{c}_{0} \eta \tilde{\xi}^{0}(\eta)+\bar{C}_{000}^{0}\left(\tilde{\xi}^{0}\right)^{3} \\
\tilde{\xi}^{k} & =-\frac{1}{\bar{\lambda}\left(k ; \kappa_{c}\right)}\left[\mathcal{B}_{k 0} \partial_{\eta} \tilde{\xi}^{0}(\eta)+a \bar{c}_{k} \eta \tilde{\xi}^{0}(\eta)+\bar{C}_{000}^{k}\left(\tilde{\xi}^{0}\right)^{3}\right]
\end{aligned}
$$

Combining the two equations in (4.17) one has

$$
\tilde{\xi}^{k}=-\frac{1}{\bar{\lambda}\left(k ; \kappa_{c}\right)}\left[\left(\mathcal{B}_{k 0}-\mathcal{B}_{00}\right) \partial_{\eta} \tilde{\xi}^{0}(\eta)+a\left(\bar{c}_{k}-\bar{c}_{0}\right) \eta \tilde{\xi}^{0}(\eta)+\left(\bar{C}_{000}^{k}-\bar{C}_{000}^{0}\right)\left(\tilde{\xi}^{0}\right)^{3}\right]
$$

We know that all the eigenvalues $\bar{\lambda}\left(k ; \kappa_{c}\right)$ are positive except the one which is zero. Since these positive eigenvalues form a continuum we can, without loss of generality, write $\lambda\left(k ; \kappa_{c}\right)=k^{2}$. This means that our expansion (4.16) is valid only if the quantities $\left(\mathcal{B}_{k 0}-\mathcal{B}_{00}\right),\left(\bar{c}_{k}-\bar{c}_{0}\right),\left(\bar{C}_{000}^{k}-\right.$ $\left.\bar{C}_{000}^{0}\right)$ go to zero at least as fast as $k^{2}$. In a way quite similar to [10] it turns out that this is indeed true - precisely when $\kappa=\kappa_{c}$. This is shown in detail for a toy model which is quite similar to our case in the appendix.

We therefore conclude that the dynamics in the critical region is again dominated by the zero mode which now satisfies a Landau-Ginsburg equation with a first order time derivative - the first equation in (4.17). This clearly yields a dissipative time evolution. The dissipation is of course due to a finite temperature and is caused by inflow into the horizon. Reverting to the original variables and noting that on the boundary $u=t$, the time of the field theory, we get a scaling solution

$$
\langle\mathcal{O}\rangle(t ; v)=v^{1 / 4}\left\langle\mathcal{O}\left(t v^{1 / 2} ; 1\right)\right\rangle
$$

This will be shown to be consistent with Kibble-Zurek scaling with $z=2, \nu=1 / 2$. 


\section{$5 \quad$ Numerical Results}

\subsection{Soliton background}

After suitable changes of variables and field redefinitions for simplification, we solved the resulting equation of motion on a Chebyshev grid using pseudo-spectral derivative method. The $k$-th lattice point on a Chebyshev grid is defined in the following way,

$$
\rho_{k}=\rho_{\star}\left(1-\cos \frac{k \pi}{N}\right)
$$

where, $N$ denotes the total number of points on the grid. At the center of the soliton we put a regularity condition on the field $\phi$.

We dealt with a specific case of the $A d S_{d+2}$ soliton, taking $d=3$ and $m^{2}=-15 / 4$ on a grid with total number of points, $N=61$. Setting the mass parameter at the conformal value simplifies the numerics. The numerical calculation of the critical exponent involves following steps :

- First we calculated $\kappa_{c}$ using the linear static equation and obtained $\kappa_{c} \approx-0.495129$.

- Next, we solved the non-linear static equation on the Chebyshev grid iteratively using a $\kappa=\kappa_{c}-a$ in the boundary condition. $a$ is an arbitrary constant chosen to be $a=0.1$.

- The above field configuration was used to specify the initial conditions at some early time $t=-t_{\max }$ in the full dynamic equation, which was solved using a time dependent $\kappa$-profile of the form $\kappa(t)=\kappa_{c}+a \tanh (v t)$. Near the phase transition point (i.e. $t=0$ ) $\kappa$ behaves linearly like $\kappa \approx \kappa_{c}+a v t$.

- This was done for various values of $v$. Using small numerical values of $v$ we expect to find the system in a scaling regime. At time $t=0$ the value of the order parameter, $\langle\mathcal{O}\rangle$ was numerically calculated from the solution and then the suitable plot [see Figure(5)] was made to check the scaling.

The above fit clearly confirms our analytical expectation, viz.,

$$
\langle\mathcal{O}(0)\rangle \sim v^{1 / 3}
$$

We also checked that changing $d \kappa$ and $N$ does not significantly change the exponent. To understand the full time dependence and the scaling of time (Eq. 3.18) one can plot the scaled response (Fig 6).

\subsection{Black Brane background}

Here we solve the PDE's by slightly different method by calculating finite difference derivative on a lattice. We choose lattice size to be npoints $=500$. The resulting discretized equations are again solved by method of lines. Near the black hole horizon we impose an ingoing 


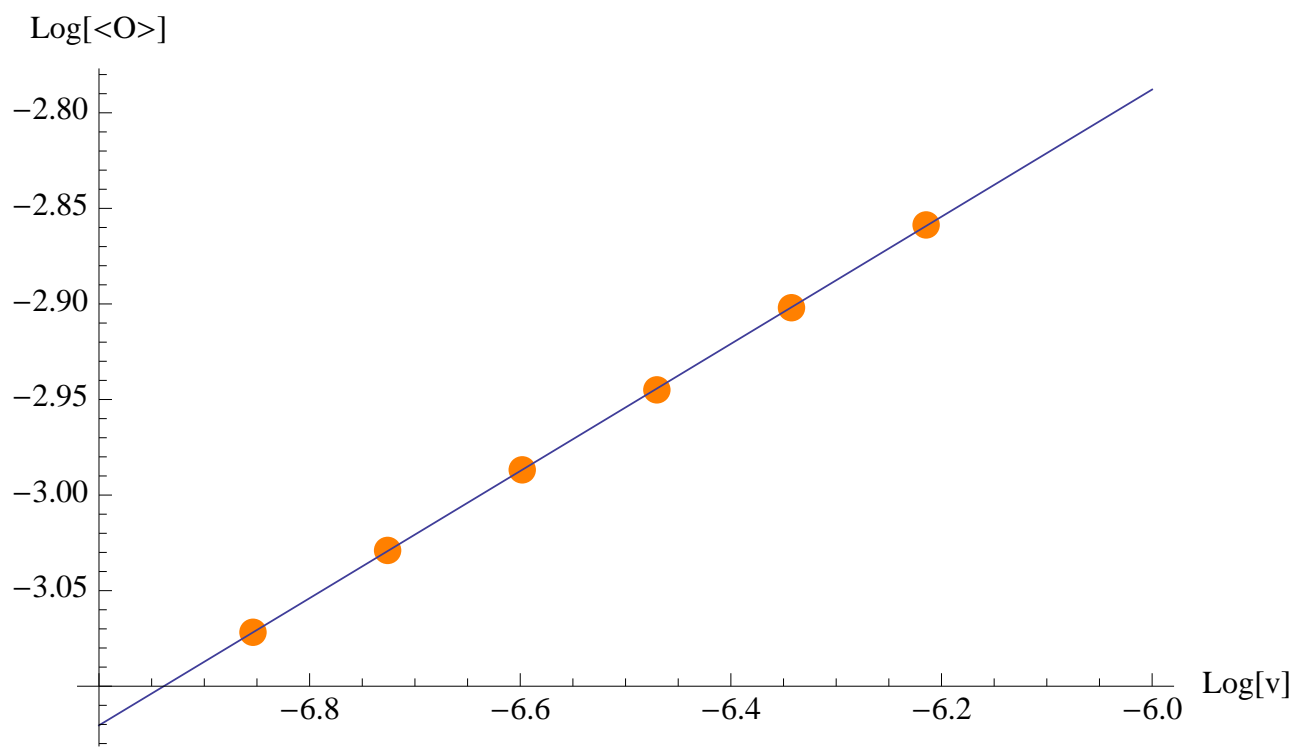

Figure 5. The scaling behavior of the order parameter $\mathcal{O}$ as a function of $v$ in in a $\phi^{4}$ theory in $A d S$ soliton geometry. The fit gives, $\ln \langle\mathcal{O}\rangle=-0.791971+0.332643 \ln v$.

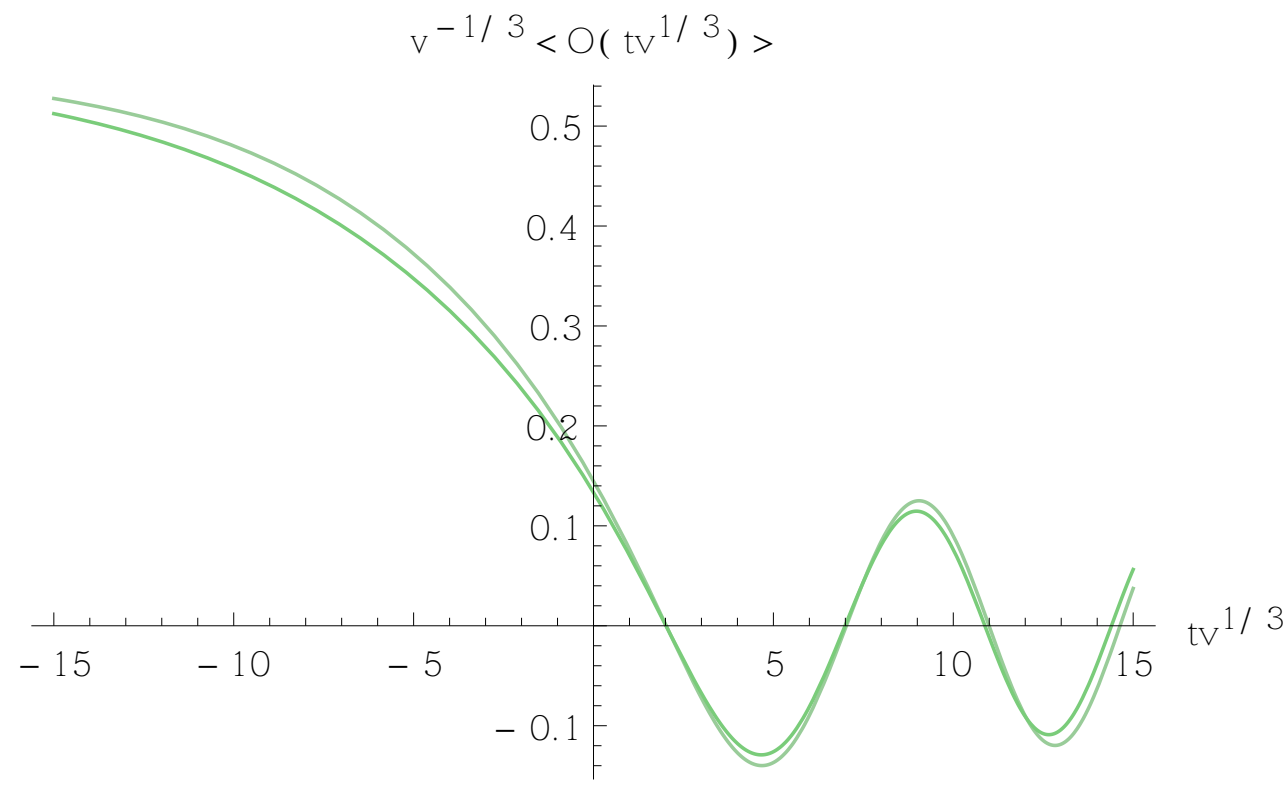

Figure 6. The scaling behavior of the order parameter $\mathcal{O}$ as a function of $t$ in in a $\phi^{4}$ theory in $A d S$ soliton geometry. Plots from the top are for $v=0.03,0.024$. These plots show scaling consistent with Eq. 3.18.

boundary condition. The main steps of the numerics, including the value of $\kappa_{c}$ and the time dependent profile $k(t)$, are identical to the soliton case : we do not repeat the details. The best fit here $[$ see Figure(7)] is given by, $\ln \langle\mathcal{O}\rangle=0.253967 \ln (v)-0.195079$ which conforms 
with our analytic result,

$$
\langle\mathcal{O}(0)\rangle \sim v^{1 / 4}
$$

Like the soliton case, we have also checked that the temporal scaling matches with Eq. 4.19.

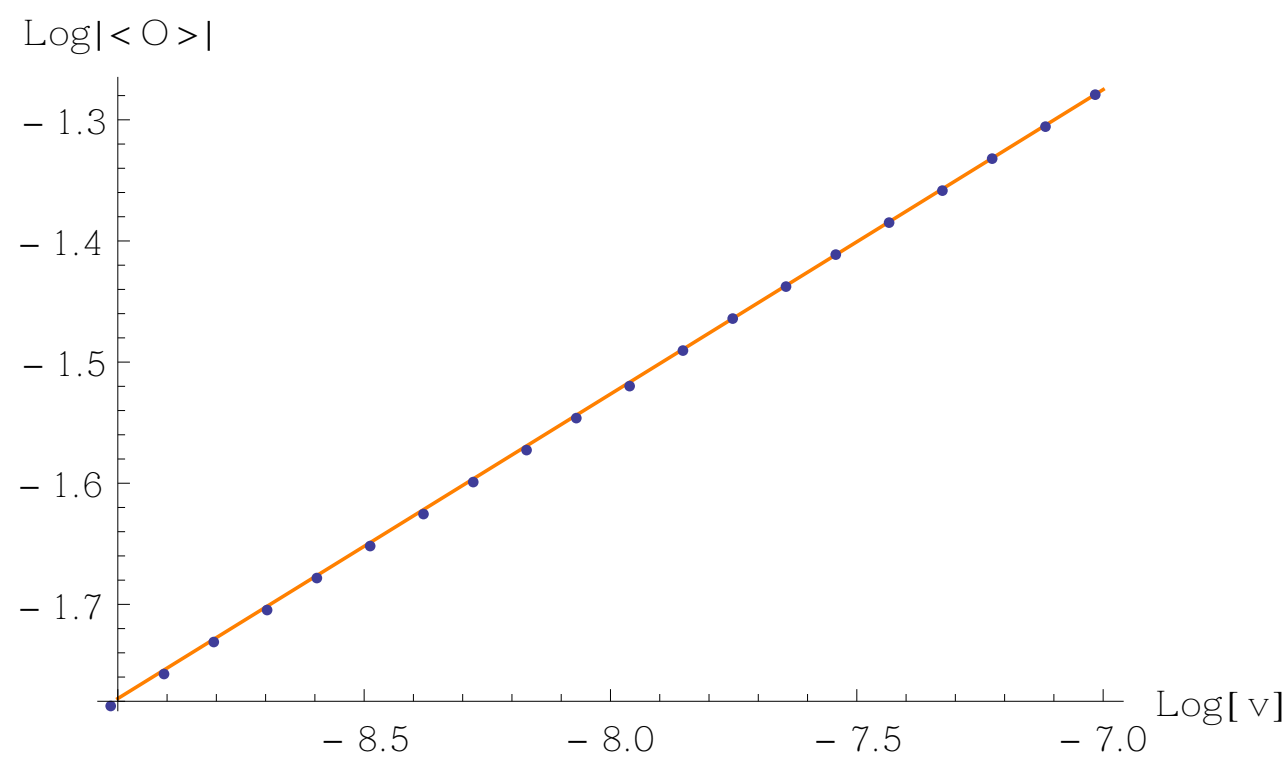

Figure 7. The scaling behavior of the order parameter $\mathcal{O}$ as a function of $v$ for a $\phi^{4}$ theory in the $A d S$ blackhole background. The fit gives, $\ln \langle\mathcal{O}\rangle=0.253967 \ln (v)-0.195079$.

In the probe approximation the late time behavior of the scalar field in black hole and soliton backgrounds are very different due to presence of the horizon in a black hole background. Any excess energy in bulk is gradually engulfed by the black hole and at very late time we have a almost static scalar profile. The late time decay of excitations of the scalar is determined by the quasi-normal modes. In a soliton background, the excess energy does not dissipate once the quenching is stopped and the scalar field shows temporal oscillation at late time. Our numerics confirm these assertions.

\section{Arbitrary exponents and Kibble-Zurek Scaling}

In this section we discuss the connection of the holographic derivation for scaling behavior in critical dynamics with the standard arguments leading to Kibble Zurek scaling.

The standard argument for Kibble-Zurek scaling for a quantum critical point proceeds as follows $[1,11]$. In the vicinity of such a transition the energy gap $\Delta$ depends on the control parameter $\lambda$ (with the critical value of $\lambda$ chosen to zero without loss of generality) as $\Delta \sim \lambda^{z \nu}$, where $z$ is the dynamical critical exponent and the $\nu$ the correlation length exponents. Consider quenching this control parameter across this transition so that near the critical point $\lambda \sim(v t)^{\alpha}$. Then the instantaneous value of the energy gap in this region is given by $\Delta_{i n s t} \sim(v t)^{\alpha z \nu}$. The criteria for the breakdown of adaibaticity during such a quench is [1] 
$d \Delta / d t \sim \Delta^{2}$. Substituting the expression for $\Delta$ and noting that the critical point is reached at $t=0$, one finds that the time spent by the system in the non-adiabatic regime is

$$
T \sim v^{-\alpha z \nu /(\alpha z \nu+1)}
$$

Next one makes the important assumption that the time evolution after the breakdown of adiabaticity is diabatic. This means that one can then argue that the order parameter is determined by the instantaneous value at time $T$. Furthermore, for slow quenches, the breakdown of adiabaticity occurs in the critical region sufficiently close to the critical point so that one can assume standard critical scaling holds. Since in this region the order parameter $\mathcal{O}$ varies with the control parameter $\lambda$ as $\mathcal{O} \sim \lambda^{\beta}$ we get

$$
\left\langle\mathcal{O}>\sim(v T)^{\alpha \beta} \sim v^{\alpha \beta /(\alpha z \nu+1)}\right.
$$

The adiabatic-diabatic assumption is rather drastic. In contrast, the holographic treatment of the present paper as well as that in [10] and [12] we derived a set of scaling relations from the properties of the solutions to the bulk equations of motion without any assumption about the nature of time evolution after breakdown of adiabaticity. The physics of the bulk is essential in this derivation, which is not at all transparent in the boundary field theory description. We will now show that the scaling relations we obtained are, nevertheless, consistent with the standard Kibble Zurek results described above.

There are several critical exponents involved in these relations. First, the static exponent $\beta$ follows from the leading nonlinearity of the bulk potential, as argued in section 2 . If the leading term in the potential is $\phi^{n+1}$ the value of $\beta$ is given by equation $(2.23), \beta=1 /(n-1)$. To find the values of $z$ and $\nu$ we need to look at the dispersion relation of small fluctuations around the static solution. The linearized fluctuations would satisfy an equation of the form

$$
\partial_{t}^{m} \delta \psi=\left[\mathcal{Q}+n F(\rho) \psi_{0}^{n-1}\right] \delta \psi
$$

where $\psi_{0}$ is the static solution, and in the examples described in this paper we have $m=$ $2, \mathcal{Q}=\mathcal{D}, F(\rho)=G(\rho)$ for the soliton and $m=1, \mathcal{Q}=\mathcal{P}, F(\rho)=\bar{G}(\bar{\rho})$ for the black hole. The control parameter is $\lambda=\left(\kappa_{c}-\kappa\right)$. The second term on the right hand side is therefore always of the order $O(\lambda)$. The dependence of the first term on $\lambda$ is determined by the nature of the background. Suppose the smallest eigenvalue of $\mathcal{Q}$ is $O\left(\lambda^{1 / p}\right)$. In both the soliton and the black hole we had $p=1$ : here we have allowed for the possibility of other backgrounds with arbitrary $p$. Then the energy of excitations $E$ is given by

$$
\begin{aligned}
& E^{m} \sim \lambda^{1 / p} \quad p>1 \\
& E^{m} \sim \lambda \quad p<1
\end{aligned}
$$

From the definition of the standard exponents we therefore have

$$
\begin{aligned}
z \nu & =\frac{1}{p m} & & p>1 \\
z \nu & =\frac{1}{m} & & p<1
\end{aligned}
$$


Now consider the condition for breakdown of adiabaticity. This again involves a solution of an equation of the form

$$
\partial_{t}^{m} \psi_{0}=\left[\mathcal{Q}+n F(\rho) \psi_{0}^{n-1}\right] \psi^{\prime}
$$

where $\psi^{\prime}$ denotes the leading correction to the adiabatic result. It is then clear that the condition $\psi^{\prime} \sim \psi_{0}$ leads to an adiabaticity breaking time $t_{a d}$

$$
\begin{aligned}
t_{a d} & \sim(v)^{-\frac{\alpha}{\alpha+p m}} & & p>1 \\
t_{a d} & \sim(v)^{-\frac{\alpha}{\alpha+m}} & & p<1
\end{aligned}
$$

With the value of $(z \nu)$ obtained in (6.5) this reproduces the relation (6.1). The instantaneous value of $\psi$, and therefore the order parameter at this time is then clearly seen to be

$$
\begin{aligned}
&\langle\mathcal{O}\rangle\left(t_{a d}\right) \sim(v)^{\frac{m \alpha}{(n-1)(\alpha+m)}}=\mathcal{O}_{a d} \quad p>1 \\
&\langle\mathcal{O}\rangle\left(t_{a d}\right) \sim(v)^{\frac{m p \alpha}{(n-1)(\alpha+m p)}}=\mathcal{O}_{a d} \quad p<1
\end{aligned}
$$

The value of $z$ requires knowledge of the way space derivatives appear in the equation of motion. In the examples we have discussed in this paper (as well as in $[10,12]$ ) the bulk equation of motion contains two space derivatives. Thus with $m$ time derivatives we have $z=2 / m$. It is then clear that (6.8) reproduces (6.2) with $\beta=1 /(n-1)$ as derived above.

Once the scalings of $t_{a d}$ and $\mathcal{O}$ are known the rescaling of fields and time required to expose the dynamics in the critical region after breakdown of adiabaticity is clear - we need to perform

$$
t \rightarrow \eta=\frac{t}{t_{a d}} \quad \psi \rightarrow \chi=\frac{\psi}{\mathcal{O}_{a d}}
$$

The analysis of sections (3.1) and (4.2) can be carried out in a straightforward fashion leading to a scaling solution

$$
\langle\mathcal{O}\rangle(t, v)=(v)^{\frac{m p \alpha}{(n-1)(\alpha+m p)}}\langle\mathcal{O}\rangle\left(t / t_{a d}, 1\right)
$$

which agrees with the Kibble-Zurek solution obtained earlier.

In the above discussion we have indicated what should be the nature of the bulk theory which leads to nontrivial values of $z$ and $\nu$. In a relativistic bulk theory, we always start with two time derivatives in the equations of motion. However the presence of a gauge field and/or a black hole horizon effectively leads to $m=1$. Values of $m \geq 3$ would be rather pathological in a bulk theory.

\section{Remarks}

As in [10] and [12] we have demonstrated the emergence of a scaling solution in the critical region in the probe approximation for a quench which is more natural from the boundary field theory point of view. The next obvious step is to study this issue with gravitational backreaction, particularly for the soliton background. Pretty much like global $A d S$ we expect that for a slow quench which does not come close to a critical point, a black hole is not formed 
immediately [29]. A black hole may, however, form a late times [30]. However near the critical point we expect that a breakdown of adiabatic evolution leads to a black hole formation at early times, and it would be interesting to look for critical behavior in this collapse. This would involve coupled partial differential equations - nevertheless we expect that the zero mode will continue to play a key role and dominate critical dynamics. In the full problem, however, it is important to consider potentials which follow from a superpotential [22, 23] so that the static solution is stable. However once again we expect that near the critical point the leading non-linearity determines the dynamics. These issues are being explored at the moment.

\section{Acknowledgements}

We would like to thank Robert Myers for a discussion. D.D. and S.R.D. would like to thank International Center for Theoretical Sciences of Tata Institute of Fundamental Research for hospitality during the final stages of this work. The work of D.D. and S.R.D. was supported in part by National Science Foundation grants NSF-PHY/0970069 and NSF-PHY/1214341.

\section{$9 \quad$ Appendix : Validity of the small $v$ expansion}

To argue for the small $v$ expansion of $\left.\bar{\xi}^{k}(\eta)(4.16)\right)$ we need to consider the eigenvalue problem

$$
\left[-\partial_{\bar{\rho}}^{2}+V_{0}(\bar{\rho})\right] \chi_{k}=k^{2} \chi_{k}
$$

The above potential $V_{0}(\bar{\rho}) \rightarrow-e^{-\bar{\rho}}$ as $\bar{\rho} \rightarrow \infty$.

The basic features of the eigenfunctions can be understood from a simpler problem in which we replace the potential by the following potential which has the same qualitative features.

$$
U(\bar{\rho})= \begin{cases}V_{0} & \text { for } \bar{\rho}=0 \\ -U_{0} & \text { for } 0 \leq \bar{\rho} \leq 1 \\ 0 & \text { for } 1 \leq \bar{\rho} \leq \infty\end{cases}
$$

with $U_{0}, V_{0}>0$. The eigenfunctions of the Schrodinger operator with eigenvalue $k^{2}>0$ are

$$
\begin{array}{ll}
\psi_{k}(\bar{\rho})=\frac{A(k)}{\sqrt{\pi}}\left(\sin \left(\sqrt{k^{2}+U_{0}} \bar{\rho}\right)+\kappa \cos \left(\sqrt{k^{2}+U_{0}} \bar{\rho}\right)\right) & 0 \leq \bar{\rho} \leq 1 \\
\psi_{k}(\bar{\rho})=\frac{1}{\sqrt{\pi}} \sin (k \bar{\rho}+\theta(\bar{\rho})) & 1 \leq \bar{\rho} \leq \infty
\end{array}
$$

Here $\kappa$ plays the role of the double trace deformation of our original problem, in the spirit that here too it dictates the modified boundary condition at $\bar{\rho}=0$. The constants $A(k)$ and 
$\theta(k)$ are determined by matching at $\bar{\rho}=1$,

$$
\begin{aligned}
& A(k)=\frac{k}{\sqrt{k^{2}\left(1+\kappa^{2}\right)+\left(\cos \left(\sqrt{k^{2}+U_{0}}\right)-\kappa \sin \left(\sqrt{k^{2}+U_{0}}\right)\right)^{2} U_{0}}} \\
& \theta(k)=\cos ^{-1}\left(\frac{\left(\cos \left(\sqrt{k^{2}+U_{0}}\right)-\kappa \sin \left(\sqrt{k^{2}+U_{0}}\right)\right) \sqrt{k^{2}+U_{0}}}{\sqrt{k^{2}\left(1+\kappa^{2}\right)+\left(\cos \left(\sqrt{k^{2}+U_{0}}\right)-\kappa \sin \left(\sqrt{k^{2}+U_{0}}\right)\right)^{2} U_{0}}}\right)-k .
\end{aligned}
$$

The solution for $k=0$ is

$$
\begin{array}{ll}
\psi_{0}(\bar{\rho})=\frac{B}{\sqrt{\pi}}\left(\sin \left(\sqrt{U_{0}} \bar{\rho}\right)+\kappa \cos \left(\sqrt{U_{0}} \bar{\rho}\right)\right) & 0 \leq \bar{\rho} \leq 1 \\
\psi_{0}(\bar{\rho})=\frac{1}{\sqrt{\pi}}(a \bar{\rho}+b) & 1 \leq \bar{\rho} \leq \infty
\end{array}
$$

The matching conditions at $\bar{\rho}=1$ now yield

$$
\begin{aligned}
B\left(\sin \left(\sqrt{U_{0}}\right)+\kappa \cos \left(\sqrt{U_{0}}\right)\right) & =a+b \\
B \sqrt{U_{0}}\left(\cos \left(\sqrt{U_{0}}\right)-\kappa \sin \left(\sqrt{U_{0}}\right)\right. & =a
\end{aligned}
$$

For any $a \neq 0$ the solution blows up at $\bar{\rho}=\infty$. Thus regular solutions require $a=0$. However the second equation in (9.6) then imply that

$$
\sqrt{U_{0}}=\cot ^{-1} \kappa
$$

These are the zero modes. In the context of our model this is the potential where we have a critical point.

The small $k$ behavior of $A(k)$ and $\theta(k)$ can be read off from the expressions (9.4). For a generic $U_{0}$ these are

$$
\begin{aligned}
& A(k) \sim \frac{k}{\left(\cos \left(\sqrt{U_{0}}\right)-\kappa \sin \left(\sqrt{U_{0}}\right) \sqrt{U_{0}}\right.}+O\left(k^{3}\right) \\
& \theta(k) \sim k\left[\frac{\kappa+\tan \sqrt{U_{0}}}{\sqrt{U_{0}}\left(1-\kappa \tan \sqrt{U_{0}}\right)}-1\right]+O\left(k^{3}\right)
\end{aligned}
$$

whereas for critical potentials we have

$$
\begin{aligned}
A(k) & \sim \frac{1}{\sqrt{1+\kappa^{2}}}\left(1-\frac{k^{2}}{8}+O\left(k^{4}\right)\right) \\
\theta(k) & \sim \frac{\pi}{2}-\frac{k}{2}
\end{aligned}
$$


This has implications for the coefficients like $\left(\mathcal{B}_{k 0}-\mathcal{B}_{00}\right)$ and $\left(\bar{C}_{k 000}-\bar{C}_{0000}\right)$ of equation(4.18). Consider the quantity, $\mathcal{B}_{k 0}$. We have,

$$
\mathcal{B}_{k 0}=\int d \bar{\rho} \bar{\chi}_{k}(\bar{\rho}) \partial_{\bar{\rho}} \bar{\chi}_{0}(\bar{\rho})
$$

If we replace the true eigenfunctions by those of our simplified problem, we get

$$
\begin{aligned}
\mathcal{B}_{k 0} & =A(k) \int_{0}^{1} d \bar{\rho}\left(\sin \left(\sqrt{k^{2}+U_{0}} \bar{\rho}\right)+\kappa \cos \left(\sqrt{k^{2}+U_{0}} \bar{\rho}\right)\right) B \sqrt{U_{0}} \\
& \times\left(\cos \left(\sqrt{U_{0}} \bar{\rho}\right)-\kappa \sin \left(\sqrt{U_{0}} \bar{\rho}\right)\right)
\end{aligned}
$$

Using (9.8) and (9.9) we therefore see that

$$
\mathcal{B}_{k 0}-\mathcal{B}_{00} \sim k \quad k \rightarrow 0
$$

for generic potentials, whereas

$$
\mathcal{B}_{k 0}-\mathcal{B}_{00} \sim k^{2} \quad k \rightarrow 0
$$

for critical potentials. The behavior for $\left(\bar{C}_{k 000}-\bar{C}_{0000}\right)$ is similar.

Going back to (4.18) we therefore see that the small $v$ expansion is generically not valid since the corrections diverge at small $k$. However for the critical potential, $\tilde{\xi}_{k}$ remain finite as $k \rightarrow 0$ and the expansion in powers of $v^{1 / 2}$ makes sense.

\section{References}

[1] For reviews and references see S. Mondal, D. Sen and K. Sengupta, Quantum Quenching, Annealing and Computation, Lecture Notes in Physics, Volume 802, arXiv:0908.2922, ; J. Dziarmaga,Advances in Physics, vol. 59, issue 6, pp. 1063-1189, arXiv:0912.4034 ; A. Polkovnikov, K. Sengupta, A. Silva and M. Vengalattore, Rev. Mod. Phys. 83, 863 (2011) [arXiv:1007.5331 [cond-mat.stat-mech]].

[2] P. Calabrese and J. L. Cardy, J. Stat. Mech. 0504 (2005) P010 [arXiv:cond-mat/0503393];

P. Calabrese and J. L. Cardy, Phys. Rev. Lett. 96 (2006) 136801 [arXiv:cond-mat/0601225];

P. Calabrese and J. Cardy, [arXiv:0704.1880 [cond-mat.stat-mech]]; S. Sotiriadis and J. Cardy, J. Stat. Mech. (2008) P11003, [arXiv:0808.0116 [cond-mat.stat-mech]]; S. Sotiriadis,

P. Calabrese and J. Cardy, EPL 87 (2009) 20002, [arXiv:0903.0895 [cond-mat.stat-mech]];

[3] J. M. Maldacena, Adv. Theor. Math. Phys. 2 (1998) 231 [Int. J. Theor. Phys. 38 (1999) 1113] [arXiv:hep-th/9711200]; S. S. Gubser, I. R. Klebanov and A. M. Polyakov, Phys. Lett. B 428, 105 (1998) [arXiv:hep-th/9802109]; E. Witten, Adv. Theor. Math. Phys. 2, 253 (1998) [arXiv:hep-th/9802150]; O. Aharony, S. S. Gubser, J. M. Maldacena, H. Ooguri and Y. Oz, Phys. Rept. 323, 183 (2000) [arXiv:hep-th/9905111].

[4] R. A. Janik and R. B. Peschanski, Phys. Rev. D 74, 046007 (2006) [arXiv:hep-th/0606149]; R. A. Janik, Phys. Rev. Lett. 98, 022302 (2007) [arXiv:hep-th/0610144]; P. M. Chesler and L. G. Yaffe, Phys. Rev. Lett. 102, 211601 (2009) [arXiv:0812.2053 [hep-th]]; P. M. Chesler, 
L. G. Yaffe, Phys. Rev. D82, 026006 (2010). [arXiv:0906.4426 [hep-th]]; S. Bhattacharyya and S. Minwalla, JHEP 0909 (2009) 034 [arXiv:0904.0464 [hep-th]]. D. Garfinkle, L. A. Pando Zayas, Phys. Rev. D84, 066006 (2011). [arXiv:1106.2339 [hep-th]]; A. Buchel, L. Lehner and S. L. Liebling, Phys. Rev. D 86, 123011 (2012) [arXiv:1210.0890 [gr-qc]].

[5] J. Abajo-Arrastia, J. Aparicio, E. Lopez, JHEP 1011, 149 (2010). [arXiv:1006.4090 [hep-th]];H. Ebrahim, M. Headrick, [arXiv:1010.5443 [hep-th]]; V. Balasubramanian, A. Bernamonti, J. de Boer, N. Copland, B. Craps, E. Keski-Vakkuri, B. Muller, A. Schafer et al., Phys. Rev. Lett. 106, 191601 (2011). [arXiv:1012.4753 [hep-th]]; V. Balasubramanian, A. Bernamonti, J. de Boer, N. Copland, B. Craps, E. Keski-Vakkuri, B. Muller, A. Schafer et al., Phys. Rev. D84, 026010 (2011). [arXiv:1103.2683 [hep-th]]; D. Galante and M. Schvellinger, JHEP 1207, 096 (2012) [arXiv:1205.1548 [hep-th]] ; E. Caceres and A. Kundu, JHEP 1209, 055 (2012) [arXiv:1205.2354 [hep-th]].

[6] T. Albash, C. V. Johnson, Electromagnetic Quenches," New J. Phys. 13, 045017 (2011). [arXiv:1008.3027 [hep-th]]; T. Takayanagi, T. Ugajin, Coarse-Graining," JHEP 1011, 054 (2010). [arXiv:1008.3439 [hep-th]]; C. T. Asplund, S. G. Avery, [arXiv:1108.2510 [hep-th]].

[7] S. R. Das, T. Nishioka, T. Takayanagi, JHEP 1007, 071 (2010). [arXiv:1005.3348 [hep-th]].

[8] A. O'Bannon, arXiv:0808.1115 [hep-th]. K. Hashimoto, N. Iizuka, T. Oka, [arXiv:1012.4463 [hep-th]]; K. -Y. Kim, J. P. Shock, J. Tarrio, JHEP 1106, 017 (2011). [arXiv:1103.4581 [hep-th]]; S. Prem Kumar, Phys. Rev. D84, 026003 (2011). [arXiv:1104.1405 [hep-th]]. S. Janiszewski, A. Karch, [arXiv:1106.4010 [hep-th]]; C. Hoyos, T. Nishioka, A. O'Bannon, [arXiv:1106.4030 [hep-th]].

[9] S. R. Das, J. Michelson, K. Narayan and S. P. Trivedi, Phys. Rev. D 74, 026002 (2006) [hep-th/0602107]; A. Awad, S. R. Das, S. Nampuri, K. Narayan and S. P. Trivedi, Phys. Rev. D 79, 046004 (2009) [arXiv:0807.1517 [hep-th]]. A. Awad, S. R. Das, A. Ghosh, J. -H. Oh and S. P. Trivedi, Phys. Rev. D 80, 126011 (2009) [arXiv:0906.3275 [hep-th]].

[10] P. Basu and S. R. Das, JHEP 1201, 103 (2012) [arXiv:1109.3909 [hep-th]].

[11] T. W. B. Kibble, J. Phys.A 9, 1387 (1976); W. H. Zurek, Nature 317, 505 (1985).

[12] P. Basu, D. Das, S. R. Das and T. Nishioka, JHEP 1303, 146 (2013) [arXiv:1211.7076 [hep-th]].

[13] K. Murata, S. Kinoshita and N. Tanahashi, JHEP 1007, 050 (2010) [arXiv:1005.0633 [hep-th]];

[14] M. J. Bhaseen, J. P. Gauntlett, B. D. Simons, J. Sonner and T. Wiseman, Phys. Rev. Lett. 110, 015301 (2013) [arXiv:1207.4194 [hep-th]].

[15] A. Buchel, L. Lehner and R. C. Myers, JHEP 1208, 049 (2012) [arXiv:1206.6785 [hep-th]]. A. Buchel, L. Lehner, R. C. Myers and A. van Niekerk, JHEP 1305, 067 (2013) [arXiv:1302.2924 [hep-th]]. A. Buchel, R. C. Myers and A. van Niekerk, arXiv:1307.4740 [hep-th].

[16] S. Sotiriadis and J. Cardy, arXiv:1002.0167 [quant-ph].

[17] S. R. Das and K. Sengupta, JHEP 1209, 072 (2012) [arXiv:1202.2458 [hep-th]].

[18] A. Chandran, A. Erez, S. Gubser and S. Sondhi, Phys. Rev. B 86, 064304 (2012); A. Chandran, A. Nanduri, S. Gubser and S. Sondhi, arXiv:1304.2402 [cond-mat.stat-mech]

[19] G. Mandal and T. Morita, arXiv:1302.0859 [hep-th]. 
[20] N. Iqbal, H. Liu, M. Mezei, Q. Si, Phys. Rev. D82, 045002 (2010). [arXiv:1003.0010 [hep-th]].

[21] T. Nishioka, S. Ryu and T. Takayanagi, JHEP 1003, 131 (2010) [arXiv:0911.0962 [hep-th]], G. T. Horowitz and B. Way, JHEP 1011, 011 (2010) [arXiv:1007.3714 [hep-th]].

[22] T. Faulkner, G. T. Horowitz and M. M. Roberts, JHEP 1104, 051 (2011) [arXiv:1008.1581 [hep-th]].

[23] T. Faulkner, G. T. Horowitz and M. M. Roberts, Class. Quant. Grav. 27, 205007 (2010) [arXiv:1006.2387 [hep-th]].

[24] I. R. Klebanov and E. Witten, Nucl. Phys. B 556, 89 (1999) [hep-th/9905104];

[25] E. Witten, hep-th/0112258; M. Berkooz, A. Sever and A. Shomer, JHEP 0205, 034 (2002) [hep-th/0112264]; A. Sever and A. Shomer, JHEP 0207, 027 (2002) [hep-th/0203168]. W. Mueck, Phys. Lett. B 531, 301 (2002) [hep-th/0201100]; S. S. Gubser and I. R. Klebanov, Nucl. Phys. B 656, 23 (2003) [hep-th/0212138]; L. Vecchi, Phys. Rev. D 82, 045013 (2010) [arXiv:1004.2063 [hep-th]]; L. Vecchi, JHEP 1104, 056 (2011) [arXiv:1005.4921 [hep-th]].

[26] S. A. Hartnoll, C. P. Herzog, G. T. Horowitz, JHEP 0812, 015 (2008). [arXiv:0810.1563 [hep-th]]; S. A. Hartnoll, C. P. Herzog, G. T. Horowitz, Phys. Rev. Lett. 101, 031601 (2008). [arXiv:0803.3295 [hep-th]].

[27] P. Basu and A. Ghosh, arXiv:1304.6349 [hep-th].

[28] G. T. Horowitz and V. E. Hubeny, Phys. Rev. D 62, 024027 (2000) [hep-th/9909056]; S. Bhattacharyya, V. EHubeny, S. Minwalla and M. Rangamani, JHEP 0802, 045 (2008) [arXiv:0712.2456 [hep-th]].

[29] S. Bhattacharyya and S. Minwalla, JHEP 0909, 034 (2009) [arXiv:0904.0464 [hep-th]].

[30] P. Bizon and A. Rostworowski, Phys. Rev. Lett. 107, 031102 (2011) [arXiv:1104.3702 [gr-qc]]; O. J. C. Dias, G. T. Horowitz, D. Marolf and J. E. Santos, Class. Quant. Grav. 29, 235019 (2012) [arXiv:1208.5772 [gr-qc]];D. Garfinkle, L. A. Pando Zayas and D. Reichmann, JHEP 1202, 119 (2012) [arXiv:1110.5823 [hep-th]].

[31] E. Kiritsis and V. Niarchos, JHEP 1208, 164 (2012) [arXiv:1205.6205 [hep-th]]. 